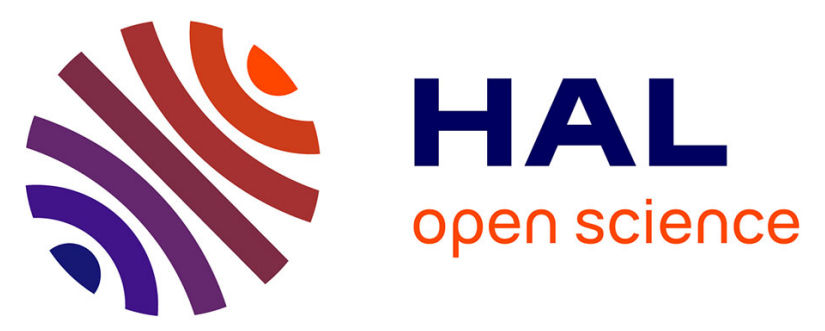

\title{
A multi-frequency Acoustic Concentration and Velocity Profiler (ACVP) for boundary layer measurements of fine-scale flow and sediment transport processes
}

David Hurther, Peter Derreck Thorne, Mickaël Bricault, Ulrich Lemmin, Jean-Marc Barnoud

\section{To cite this version:}

David Hurther, Peter Derreck Thorne, Mickaël Bricault, Ulrich Lemmin, Jean-Marc Barnoud. A multi-frequency Acoustic Concentration and Velocity Profiler (ACVP) for boundary layer measurements of fine-scale flow and sediment transport processes. Coastal Engineering, 2011, 58, pp.594-605. 10.1016/j.coastaleng.2011.01.006 . hal-00614448

\section{HAL Id: hal-00614448 \\ https://hal.science/hal-00614448}

Submitted on 29 Sep 2021

HAL is a multi-disciplinary open access archive for the deposit and dissemination of scientific research documents, whether they are published or not. The documents may come from teaching and research institutions in France or abroad, or from public or private research centers.
L'archive ouverte pluridisciplinaire HAL, est destinée au dépôt et à la diffusion de documents scientifiques de niveau recherche, publiés ou non, émanant des établissements d'enseignement et de recherche français ou étrangers, des laboratoires publics ou privés. 
Provided for non-commercial research and education use. Not for reproduction, distribution or commercial use.

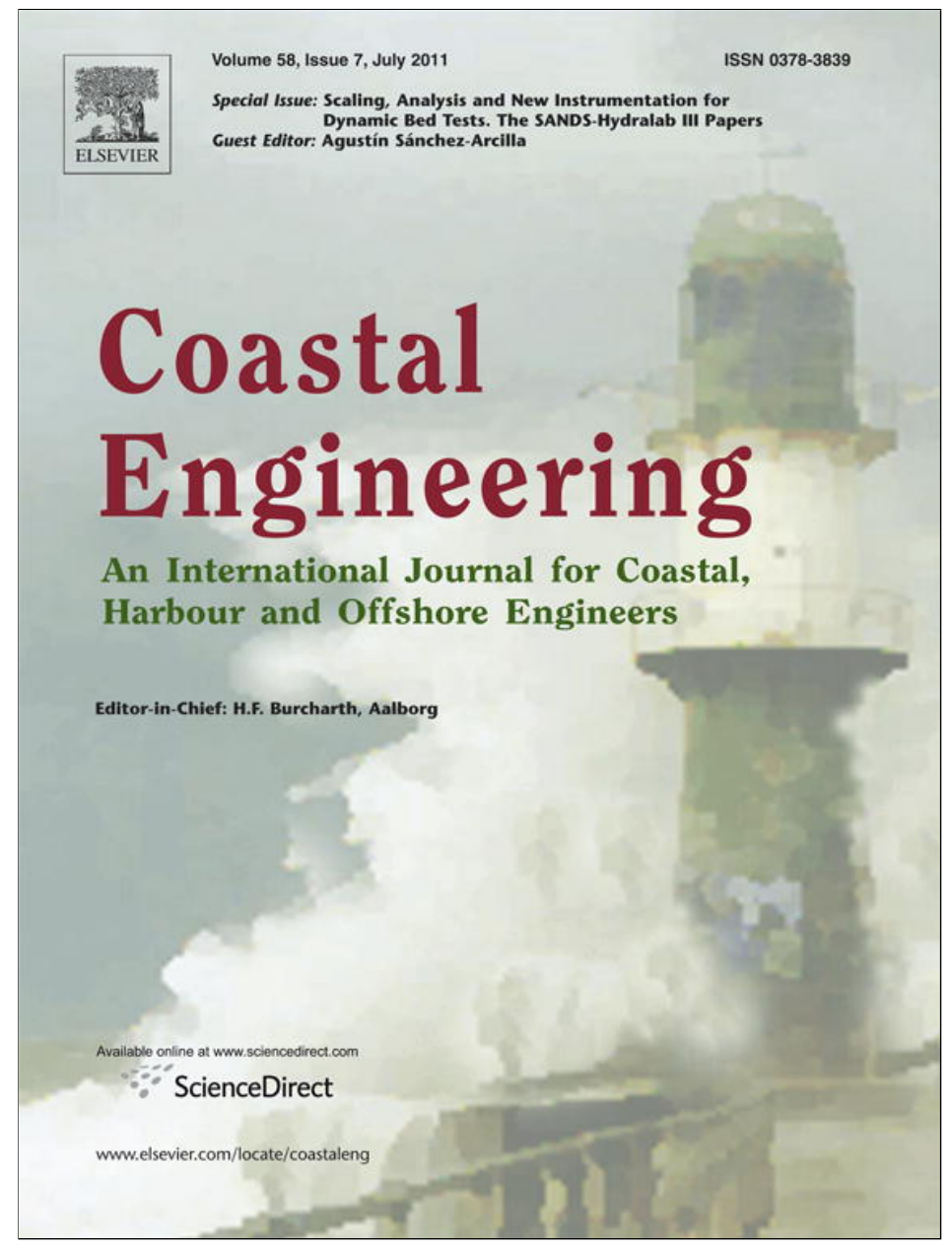

This article appeared in a journal published by Elsevier. The attached copy is furnished to the author for internal non-commercial research and education use, including for instruction at the authors institution and sharing with colleagues.

Other uses, including reproduction and distribution, or selling or licensing copies, or posting to personal, institutional or third party websites are prohibited.

In most cases authors are permitted to post their version of the article (e.g. in Word or Tex form) to their personal website or institutional repository. Authors requiring further information regarding Elsevier's archiving and manuscript policies are encouraged to visit:

http://www.elsevier.com/copyright 


\title{
A multi-frequency Acoustic Concentration and Velocity Profiler (ACVP) for boundary layer measurements of fine-scale flow and sediment transport processes
}

\author{
David Hurther ${ }^{\mathrm{a}, *}$, Peter D. Thorne ${ }^{\mathrm{b}}$, Mickaël Bricault ${ }^{\mathrm{a}}$, Ulrich Lemmin ${ }^{\mathrm{c}}$, Jean-Marc Barnoud ${ }^{\mathrm{a}}$ \\ a Laboratory of Geophysical and Industrial Flows (LEGI), CNRS-UJF-GINP, Grenoble, France \\ ${ }^{\mathrm{b}}$ National Oceanography Centre, Joseph Proudman Building, 6 Brownlow Street, Liverpool, L3 5DA, UK \\ ${ }^{\mathrm{c}}$ Laboratory of Environmental Hydraulics, Ecole Polytechnique Fédérale de Lausanne, Lausanne, Switzerland
}

\section{A R T I C L E I N F O}

Available online 2 March 2011

\section{Keywords:}

Acoustic backscattering system

Acoustic Doppler velocity profiler

Sediment transport

Coastal nearshore and surfzone

measurements

Ripple vortex entrainment

Wave breaking

\begin{abstract}
A B S T R A C T
The use of acoustics to measure sediment transport boundary layer processes has gained increasing acceptance over the past two decades. This has occurred through the development of increasingly sophisticated measuring systems and theoretical developments, which have enabled flow and suspended sediment parameters to be obtained from acoustic data with a high degree of accuracy. Until relatively recently, separate acoustic systems were used to measure flow and suspended sediment concentration. Over the past few years, however, the technology has become sufficiently advanced so that flow and sediment measurements can be integrated into a single system. This integration provides, quasi-instantaneous, non-intrusive, co-located, high temporal-spatial resolution measurements of benthic flow and sediment processes. Here the development of such an instrument, the Acoustic Concentration and Velocity Profiler (ACVP) is described. The theory underpinning its application is outlined, new approaches to velocity de-aliasing and suspended sediment inversion instabilities using multi-frequency capabilities are presented and the application of the system to sediment transport processes over a sandy ripple bed is illustrated. The observations clearly show the value of such instrumentation for studying the dynamical interaction between the bed, the flow and the sediments at and within the bottom boundary layer.
\end{abstract}

(c) 2011 Elsevier B.V. All rights reserved.

\section{Introduction}

Over the past twenty years, a variety of Acoustic Backscattering Systems (ABS) have been developed and used intensively for the study of natural, anthropised or manmade hydrosystems. Long range ABS operating in the $10 \mathrm{KHz}$ to $100 \mathrm{KHz}$ acoustic frequency are capable of profiling currents and backscatter characteristics of suspended matter (sediments, fish, plankton, air bubbles) over distances of more than several hundred meters. They are applied to study large scale phenomena such as ocean circulation, internal waves, river basins and lake dynamics, as well as coastal, nearshore and estuarine dynamics. High-resolution ABS working in the $\mathrm{MHz}$ range using point measurements or profiling systems usually cover distances between $5 \mathrm{~cm}$ to $1 \mathrm{~m}$ from the emitting transducer with a spatial resolution in the $\mathrm{mm}$ to $\mathrm{cm}$ range. These systems are well suited for flow/sediment/bedform process studies across the active benthic flow region with a minimum flow perturbation effect from the ABS. As outlined in Thorne and Hanes (2002), these techniques provide measurements of the physical quantities relevant to sediment transport. They defined the quantities as a measurement triad

\footnotetext{
* Corresponding author.

E-mail address: david.hurther@hmg.inpg.fr (D. Hurther).
}

composed of the nearbed flow quantities (velocity, shear, turbulence), the bedform parameters (bed constitution, position, shape and deformation) and the suspension quantities (sediment concentration, erosion and accretion fluxes, sediment size). All three components can interact dynamically with feedback processes occurring; these complex boundary layer interactions explain why our understanding of sediment transport processes is still limited and our long term predictive capability of sediment transport uncertain.

The present study reports on the development of an Acoustic Concentration and Velocity Profiler, ACVP, which was carried out within the European project HYDRALAB III as a Joint Research Activity on Scaling Analysis and New instrumentation for mobile beD testS (SANDS). The ACVP is capable of co-located high resolution profiling of the multi-component flow velocity and suspended sediment concentration, thus providing direct sediment flux measurements across the benthic flow region. Sediment flux profiling at intrawave and turbulent scale above a mobile sediment bed is recognized as one of the most promising and challenging aspects in sediment transport process studies. To-date velocity and concentration measurements were generally collected using separate instruments (Harris et al., 2003; VanderWerf et al., 2007; Thorne et al., 2009; O'Hara Murray et al., 2011). This provided observations that were not co-located and thus limited the investigation of sediment fluxes to intermediate scale processes (of the order of decimetres). In particular, turbulence 
processes which are one of the leading sediment entrainment mechanisms could not be directly addressed for flow scales smaller than the separation distance between the instruments. These colocated high-resolution measurements are essential to many sediment transport processes such as ripple vortex entrainment of sand, sediment processes in the surf and swash zones, fine sediment transport processes over large-scale gravel beds, cohesive sediment transport processes, and sand/mud mixtures erosion processes.

Few ACVP instruments have been developed in the past. Shen and Lemmin (1999) designed a system using simultaneously back and forward scattering for concentration measurements and co-located multi-bistatic pulse coherent velocity measurements. The system gave good results in terms of concentration range profiling up to $140 \mathrm{~kg} / \mathrm{m}^{3}$ over a distance of more than a decimetre above a channel bed. In order to work, the system configuration required the alignment of two emitting/receiving transducers mounted face-toface. This configuration considerably limits its use for profiling above mobile beds without disturbing it since one of the transducers must be mounted at bed level. The spatial resolution was $6 \mathrm{~mm}$ with a temporal resolution in the $100 \mathrm{~ms}$ range. Zedel and Hay (1999) and Smyth et al. (2002) also demonstrated the possibility of co-located velocity and concentration profiling with similar performances in terms of spatio-temporal resolution.

The objective here is to report on significant improvements in ACVP performance in terms of measurement accuracy, resolution, range (velocity and concentration) and observational capability using a co-located multi-frequency Doppler system. Several methods are proposed and tested to overcome the velocity and concentration limitations due to Doppler phase aliasing and inversion instabilities due to the sediment attenuation effect on the backscattered intensity profile. As a result, the present high resolution ACVP is particularly well adapted to sediment flux measurement over a wide range of flow scales in the challenging benthic flow region.

In the following Section 2, we will present the basic principles of multi-bistatic pulse-coherent velocity profiling and incoherent backscattered intensity inversion for sediment concentration profiling. Section 3 is devoted to a new Doppler velocity de-aliasing method to overcome the limitations due to the well-known depth-velocity ambiguity relation of pulse coherent systems. In Section 4, we present a novel dual-frequency (also called bi-frequency) inversion method generating stable sediment concentration profiles across the highconcentration nearbed flow region. In Section 5, the performance of the ACVP is tested for velocity, sediment concentration and sediment flux profiling above a rippled sand bed under a regular wave forcing.

\section{ABS principles for combined profiling of velocity and suspended sediment concentration}

The basic principles used for quasi-instantaneous, simultaneous and co-located profiling of velocity and sediment concentration can be explained from the expression of the scattered pressure at the face of a receiving piezo-electrical transducer $\mathrm{R}$ located at a distance $r_{k, r}$ from a suspended particle $k$ in the farfield of the transducer (Fig. 1):

$p=A\left(r_{k, i}, r_{k, r}, a_{p}, \theta\right) \exp \left\{i\left[\omega_{c} t+k_{c}\left(r_{k, i}+r_{k, r}\right)\right]\right\}$

with

$A\left(r_{k, i}, r_{k, r}, a_{p}, \theta\right)=\frac{p_{0} r_{0}}{2} a_{p} f_{p}\left(\theta, a_{p}\right) \frac{D_{i} D_{r}}{r_{k, i} r_{k, r}} \exp \left[-\alpha\left(r_{k, i}+r_{k, r}\right)\right]$

Particle $k$ is within a cloud of $N$ suspended particles insonified by an incident pulse train transmitted at the pulse repetition frequency, $P R F$, by a transmitting transducer $\mathrm{T}$ located at distance $r_{k, i}$ from particle $k . \omega_{c}$ and $k_{c}$ are the angular frequency and wave number of the transmitted signal, respectively. $a_{p}$ and $\theta$ are the particle radius and

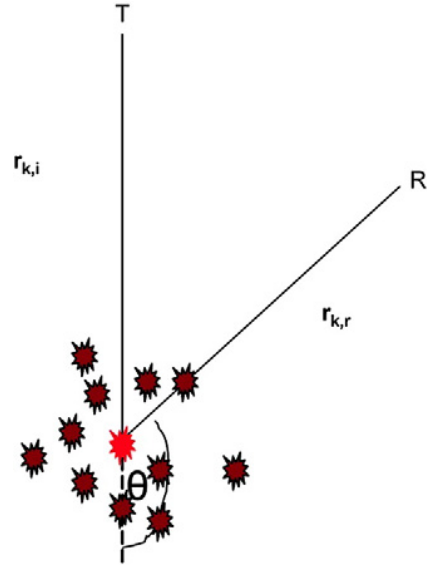

Fig. 1. Suspended sediment cloud insonified by an ABS in bistatic configuration.

the backscattering angle (equal to $\pi$ when the transmitter is used as a receiver and transceiver, i.e. in monostatic configuration). $p_{0}$ is the reference pressure at distance $r_{0}$ from the transmitter. $f_{p}, D_{i}$, and $D_{r}$ are the particle form function and directivity functions of the transmitter and receiver, respectively. $\alpha$ is the total pressure attenuation factor due to absorption in water and scattering losses due to suspended particles distributed along the travel path of the radiated sound.

When a large number $N$ of particles are randomly distributed over the insonified volume, the received pressure can be written as

$\hat{p}=\Omega \exp (i \gamma) \exp \left[i k_{c}\left(\hat{r}_{i}+\hat{r}_{r}\right)\right]$

where $\hat{p}$ has been demodulated in frequency. $\hat{\mathrm{r}}_{i}$ and $\hat{\mathrm{r}}_{r}$ are the mean cloud displacements spatially averaged over the insonified sample volume. Under incoherent scattering and negligible multiple scattering conditions, amplitude $\Omega$ and phase noise $\gamma$ can be expressed as

$$
\left\{\begin{array}{l}
\Omega^{2}=N \iiint_{\vartheta} A^{2} d \vartheta \\
\gamma=\arctan \left[\frac{-\sum_{k=1}^{N} A\left(r_{k, i}, r_{k, r}\right) \sin \left(\varphi_{k}\right)}{\sum_{k=1}^{N} A\left(r_{k, i}, r_{k, r}\right) \cos \left(\varphi_{k}\right)}\right] \text { with } \varphi_{k}=k_{c}\left(r_{0 k, i}+\tilde{r}_{k, i}+r_{0 k, r}+\tilde{r}_{k, r}\right)
\end{array}\right.
$$

where $r_{0 k, i}$ and $r_{0 k, r}$ correspond to the initial positions of the $k$ th particle relative to the transmitter and the receiver, respectively. $\tilde{\mathrm{r}}_{k, i}$ and $\tilde{\mathrm{r}}_{k, r}$ are the displacement deviations of the $k$ th particle from the spatially averaged displacements $\hat{\mathrm{r}}_{i}$ and $\hat{\mathrm{r}}_{r}$, respectively.

In order to measure the particle velocity and concentration in the same sample volume, both the squared amplitude $\Omega^{2}$ and the Doppler phase $k_{c}\left(\hat{r}_{i}+\hat{r}_{r}\right)+\gamma$ of the scattered pressure are acquired and treated as detailed below. The term $\gamma$ is an undesired phase noise term contributing to noise in the velocity signal.

\subsection{Velocity profiling}

For velocity profiling in the bottom metre above the bed, the acoustic pulse is typically transmitted at a PRF about one $\mathrm{kHz}$. In order to obtain a spatial resolution in the $\mathrm{mm}$ range, the acoustic pulse has a typical duration of several $\mu$ s and a carrier frequency in the $\mathrm{MHz}$ range. The demodulated complex Doppler signal at the output of the velocity system is proportional to the received pressure given in Eq. (2) as

$e(t)=I(t)+i Q(t) \propto \hat{p}(t)$

$R_{e e}(\delta)=\frac{1}{T} \int_{0}^{\infty} e(t) e(t-\delta) d t$ 
where $R_{e e}(\delta)$ is the complex autocorrelation function of $e(t)$. Note that for simplicity in the writing, signal $e(t)$ is represented as a time continuous signal even though it is inherently sampled at frequency PRF. The measurement limitations linked to this inherent time discretization are discussed in Section 3.

The phase of the complex autocorrelation signal at $\delta=T_{P R F}$ is obtained using the pulse-pair algorithm (Lhermitte and Serafin, 1984),

$$
\begin{aligned}
\Psi^{T_{P R F}} & =\arg \left[R_{e e}\left(\delta=T_{P R F}\right)\right]=\arctan \left(\frac{\operatorname{Im}\left[R_{e e}\left(\delta=T_{P R F}\right)\right]}{\operatorname{Re}\left[R_{e e}\left(\delta=T_{P R F}\right)\right]}\right) \\
& =\arctan \left(\left\langle\frac{I^{t} Q^{t-T_{P R F}}-I^{t-T_{P R F}} Q^{t}}{I^{t} I^{t-T_{P R F}}-Q^{t} Q^{t-T_{P R F}}}\right\rangle_{N p p}\right)
\end{aligned}
$$

where the last expression on RHS has been averaged over $N_{p p}$ consecutive pulse-pairs in order to limit the noise contribution in the quasi-instantaneous phase $\Psi_{\text {PRF. }}^{T_{\text {PR }}}$ For typical flow applications, $N_{p p}$ varies between 8 and 64 resulting in a final velocity time resolution of $\mathrm{PRF} / \mathrm{N}_{p p}$. Typical values for this last parameter vary between $10 \mathrm{~ms}$ and $100 \mathrm{~ms}$

When several receivers are used simultaneously in different flow directions (i.e. in a multi-bistatic configuration) to perform multicomponent velocity profiling, the quasi-instantaneous Doppler frequency for each receiver $j$ is obtained from the previous expression

$f_{D, j}=\left.\frac{1}{2 \pi} \frac{d \Psi_{j}}{d \delta}\right|_{\delta=0}=\frac{\Psi_{j}^{T_{P R F}}-\Psi_{j}^{0}}{2 \pi T_{P R F}}=\frac{\Psi_{j}^{T_{\text {PRF }}}}{2 \pi T_{P R F}}$

where $\Psi^{0}=0$ by definition. The corresponding radial velocity is equal to

$$
\begin{aligned}
v_{j}(t) & =\frac{c}{f_{c}} f_{D, j}=\vec{V} \cdot\left(\vec{e}_{i}+\vec{e}_{s, j}\right)=\frac{c k_{c}}{2 \pi f_{c}} \frac{d}{d t}\left[\hat{r}_{j}^{t}+\gamma_{j}\right] \\
& =\hat{v}_{j}(t)+n_{j}(t) \text { with } \hat{r}_{j}^{t}=\hat{r}_{i, j}+\hat{r}_{r, j}
\end{aligned}
$$

where $\vec{V}_{j}(t)$ corresponds to the radial velocity spatially averaged over the sample volume and $n_{j}(t)$ represents the velocity noise due to the Doppler phase noise contribution $\gamma_{j}$ in Eq. (3). $f_{c}$ and $c$ are the carrier frequency and the speed of sound in water, respectively.

When three receivers are used in three different directions, the profile of the quasi-instantaneous velocity vector $\vec{V}(u, v, w)$ can be reconstructed using the geometrical transformation matrix $[T]$,

$$
\left(\begin{array}{l}
u \\
v \\
w
\end{array}\right)=[T]\left(\begin{array}{l}
v_{1} \\
v_{2} \\
v_{3}
\end{array}\right)
$$

$[T]$ depends upon the arrangement of the receivers $R_{\mathrm{j}}$ relative to the central transmitter $\mathrm{T}$ (see Fig. $2 \mathrm{~b}$ ). The time averaged velocities are not affected by the Doppler noise contribution due to its fairly white noise characteristics over the resolved bandwidth (Hurther and Lemmin, 2001). However, the measured turbulent velocity variance for each radial component $j$ is affected by the additional noise variance as

$\overline{v_{j}^{\prime 2}}=\overline{\hat{v}_{j}^{\prime 2}}+\overline{n_{j}^{2}}$

with $\sigma_{j}^{2}=\overline{n_{j}^{2}}=\int_{-\infty}^{+\infty} \int_{-\infty}^{+\infty} R_{n_{j} n_{j}}(\delta) \exp (-i 2 \pi f \delta) d \delta d f$

The prime represents the time fluctuating component obtained after application of the Reynolds decomposition (i.e. $v_{j}(x, y, z, t)=$ $\bar{v}_{j}(x, y, z)+v_{j}^{\prime}(x, y, z, t)$; the overbar denotes the time averaged velocity). The noise variance in Eq. (9) is expressed as the noise spectrum integrated over the resolved bandwidth. Using the
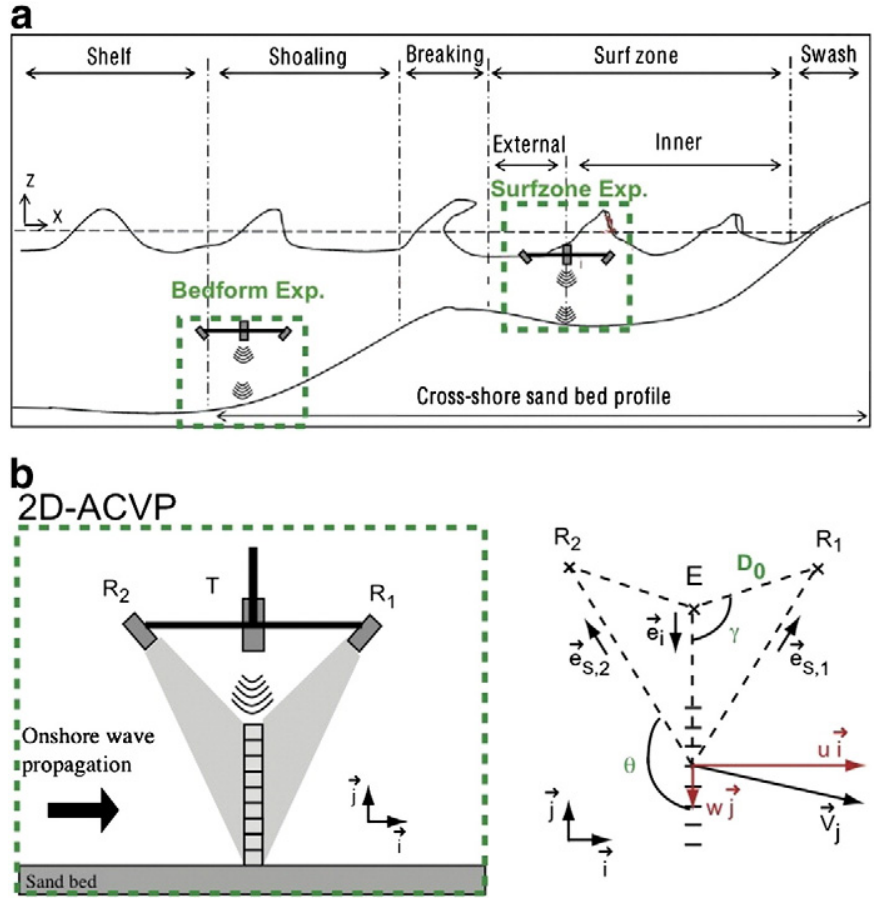

Fig. 2. (a) Typical ACVP deployments for measurements in the surf zone and in the rippled bed zone. (b) Configuration of the 2D-ACVP using one transceiver T (receiving mode is used for the concentration profiling) and 2 bistatic receivers $R_{1}$ and $R_{2}$.

geometrical transformation matrix, the noise contributions in the turbulent intensities can be expressed as (Hurther and Lemmin, 2001)

$$
\left(\begin{array}{l}
\overline{\frac{u^{\prime 2}}{v^{\prime 2}}} \\
\frac{{w^{\prime}}^{2}}{2}
\end{array}\right)=\left(\begin{array}{l}
\overline{{\hat{u^{\prime}}}^{2}} \\
\frac{{\hat{v^{\prime}}}^{2}}{{\hat{w^{\prime}}}^{2}}
\end{array}\right)+[T]^{2}\left(\begin{array}{l}
\sigma_{1}^{2} \\
\sigma_{2}^{2} \\
\sigma_{3}^{2}
\end{array}\right)
$$

The noise variances contained in the second term on the RHS of Eq. (10) can cause relative errors of up to $80 \%$ depending on the geometrical weighting factors contained in the transformation matrix and the degree of isotropy of the studied turbulence. Different Doppler noise suppression methods have been proposed to minimize these effects. In Hurther and Lemmin (2001) and Blanckaert and Lemmin (2006), the use of a fourth receiver instead of three, allows the calculation of two vertical velocity components from separate independent radial velocity components measured in different directions. The uncorrelated Doppler noises permit the use of cross correlation techniques to eliminate the additional noise variances in the reconstructed velocity components of Eq. (10). More recently, Hurther and Lemmin (2008) proposed a cross-correlation technique using the quasi-instantaneous and co-located dual-frequency capability of the present ACVP. In this case, the velocity redundancy is obtained from the measurement of the same radial velocity components at two slightly different carrier frequencies. The advantage of this method over the one of Hurther and Lemmin (2001) is the use of a single transducer at two different frequencies, thus avoiding the use of an additional receiver and corresponding hardware channels in the system. Typically, these noise reduction methods reduce the relative errors in the second and third order turbulent velocity moments to values between $10 \%$ and $30 \%$, respectively. Detailed analysis of turbulent kinetic energy budgets and coherent flow structure dynamics can then be carried out in complex turbulent flows such as rough-bed channel flows (Hurther et al., 2007a; Mignot et al., 2009a,b), or under nearshore breaking 
waves in the surfzone (Hurther et al., 2007b; Chassagneux and Hurther, 2009; Mignot et al., 2009c).

\subsection{Sediment concentration profiling}

Starting with Eqs. (1) to (3), the mean squared amplitude of the pressure (proportional to the acoustic intensity) scattered by a cloud of $N$ suspended particles in the sample volume $d V$ is written as

$$
\begin{aligned}
\hat{p}^{2}=\Omega^{2}= & \frac{3\left(p_{0} r_{0}\right)^{2}}{16 \pi}\left(\frac{\int_{0}^{\infty} a_{p}^{2}\left|f_{p}\left(\theta, a_{p}\right)\right|^{2} n\left(a_{p}\right) d a_{p}}{\rho_{p} \int_{0}^{\infty} a_{p}^{3} n\left(a_{p}\right) d a_{p}}\right) C \iiint_{V}\left(\frac{D_{i} D_{r}}{\hat{r}_{i} \hat{r}_{r}}\right)^{2} \\
& \times \exp \left[-2 \alpha\left(\hat{r}_{i}+\hat{r}_{r}\right)\right] d V
\end{aligned}
$$

where $C=N \rho_{p} \frac{4}{3} \pi \int_{0}^{\infty} a_{p}^{3} n\left(a_{p}\right) d a_{p}$ is the mass concentration and $n\left(a_{p}\right)$ corresponds to the number size distribution of the suspension cloud in $d V$. As previously mentioned, this expression is valid under incoherent backscattering and negligible multiple scattering conditions.

For the case of a sand suspension insonified by an ABS in a monostatic configuration (i.e. when the transmitter is also used as a receiver), the calibration terms in Eq. (11) have been evaluated accurately over the past twenty years (Hay and Mercer, 1985; Sheng and Hay, 1988; Hay, 1991; Thorne and Campbell, 1992; Thorne and Hanes, 2002). Based on semi-theoretical and experimental determinations of the acoustical backscattering and attenuation properties of sand suspensions, as well as appropriate calibration procedures for the estimation of the hardware parameters (Bricault, 2006; Betteridge et al., 2008), the output mean squared voltage signal (which is proportional to the mean squared pressure) of the ACVP becomes:

$I_{m o}=A_{m o} A_{s} C \exp \left(-4 \int_{0}^{\hat{r}} \zeta_{s} C d \hat{r}\right)$

where

$\left\{\begin{array}{l}A_{m o}=\left(\frac{R T_{V} p_{0} r_{0}}{1.05 k_{c} a_{t} \psi \hat{r}}\right)^{2} \frac{\tau c}{4} \exp \left(-4 \alpha_{w} \hat{r}\right) \\ A_{s}=\frac{3}{4 \rho_{s}} \frac{\left\langle a_{s}^{2} f^{2}(\theta=\pi, x)\right\rangle}{\left\langle a_{s}^{3}\right\rangle} \quad \text { with } f(\theta=\pi, x)=\left\{\begin{array}{l}g(x)\left\langle f_{\text {sphere }}(\theta=\pi, x)\right\rangle \text { theor } \\ 1.1 C_{0}(x) \frac{K_{f} x^{2}}{1+K_{f} x^{2}} \text { empir }\end{array}\right. \\ \zeta_{s}=\frac{3}{4 \rho_{s}} \frac{\left\langle a_{s}^{2} \chi\right\rangle}{\left\langle a_{s}^{3}\right\rangle} \quad \text { with } \chi=\left\{\begin{array}{l}g(x)\left\langle\chi_{\text {sphere }}(x)\right\rangle \text { theor } \\ \frac{\frac{4}{3} K_{\alpha} x^{4}}{1+x^{2}+\frac{4}{3} K_{\alpha} x^{4}} \text { empir }\end{array}\right.\end{array}\right.$

with $g(x)=\frac{\beta x^{3}+0.5 x+3.5}{x^{3}+3.5} ; \beta=1.7 \pm 0.3$ and $x=k a_{s}$.

The term $A_{m o}$ includes the system dependent parameters and the water absorption term along the profile. $R T_{V}$ is a system constant valid for the transducer sensitivity and the transfer function of the hardware unit, $a_{t}$, is the radius of the transducer, $\psi$ represents the correction function accounting for the departure from spherical spreading in the nearfield of the transducer ( $\psi=1$ in the farfield), $\tau$ is the duration of the transmitted pulse, $\alpha_{w}$ is the water attenuation constant for pressure, $a_{s}$ is the sand grain radius for a $1 / 4-\varphi$ narrow class size constituting the size distribution $n\left(a_{s}\right), \rho_{s}$ is the density of the sand particles. The term $A_{s}$ is the sand backscattering term, i.e. for $\theta=\pi$. The brackets denote averaging weighted by the size distribution of the sand suspension as written in Eq. (11) for the angular scattering term in parenthesis. The functions $f(\theta=\pi, x)$ and $\chi$ in Eq. (13) are the fundamental backscattering form function and the normalized total scattering cross section, respectively. These acoustic properties are valid for a sand suspension of nominally single size having a $1 / 4-\varphi$ number size distribution. Two different approaches for their approximations are proposed in the literature. Thorne and Buckingham (2004) have shown that they can be accurately approximated by the rigid sphere solutions that have been low-pass filtered and modulated by an empirical function $g(x)$. The low-pass filter eliminates the resonance effects in the sphere solution. The empirical modulation function accounts for the higher scattering cross section of irregularly shaped sand particles compared to spheres. Spherical shapes have the smallest ratio of surface area to volume. $\beta=1.7 \pm 0.3$ in $g(x)$ is an average value found over a large set of marine sand suspensions with strong differences in grain shape irregularities.

The second approach relies on two generic empirical approximations that have been fitted to a large set of calibration results using many different marine sand suspensions. The value of $K_{f}$ for the empirical form function is found to be equal to 1.1 and the value of $C_{0}$ tends towards unity as $x$ tends to zero and infinity. For the normalized total scattering cross section, a value of $K_{\alpha}=0.18$ has been proposed in Thorne and Hanes (2002). The generic formulations have recently been revisited by Thorne and Meral (2008).

The inverse problem defined herein consists in the determination of the unknown $C$ in Eq. (12) when the parameters $A_{m o} f(\theta=\pi, x), \chi$ and the size distribution $n\left(a_{s}\right)$ are known a priori. The performances and limitations associated with the standard inversion methods known as the iterative implicit approach (Thorne et al., 1993) and the explicit approach (Lee and Hanes, 1995) will be compared herein to a novel dual-frequency inversion method proposed below. In particular, the sensitivity to the uncertainties in the inversion parameters of the different methods will be discussed.

Please note that in many flow applications, both the variables $C$ and $\left\langle a_{s}>\right.$ are defined as unknowns in the inverse problem (Thorne and Hanes, 2002). In this case, the equation is solved by the use of the iterative implicit approach at two or three different acoustic frequencies following the method proposed in Thorne and Hardcastle (1997). However, this method assumes the sand size distribution to be of a given type (usually gaussian or log-normal) with a fixed relative width (ratio of standard deviation to mean value). In the following, only $C$ is defined as the unknown variable to be determined.

\section{A versatile velocity de-aliasing technique}

One of the most limiting aspects of pulsed coherent Doppler velocity techniques resides in its depth-to-velocity ambiguity relation. It is the consequence of the pulsed transmitting mode leading to an inherent time discretization at $T_{P R F}=1 / P R F$ of the Doppler frequency $f_{D, j}\left(n T_{P R F}\right)$. Applying Shannon's sampling theorem, the maximal unaliased Doppler frequency is limited to the value $P R F / 2$. Consequently the maximal profiling depth $L_{\max }$ is limited by the period $T_{P R F}$ as follows:

$$
\left\{\begin{array}{l}
v_{j, \text { max }}=\frac{c}{2 f_{c} T_{P R F}} \\
L_{\text {max }}=\frac{D_{0}-\left(c T_{P R F}\right)^{2}}{2\left(D_{0} \cos \gamma-c T_{P R F}\right)}
\end{array}\right.
$$

$D_{0}$ represents the distance between the transmitter and the receiver in a bistatic configuration (see Fig. 2b). In a monostatic 
configuration $\left(D_{0}=0\right)$, the well known ambiguity relation between the maximal profiling depth and the maximal velocity becomes

$v_{j, \max }=\frac{c^{2}}{4 f_{c} L_{\max }}$

Here $\nu_{j, \max }$ is the radial velocity component expressed in Eq. (7). In the monostatic configuration it corresponds to twice the projection of the velocity onto the transducer axis.

For applications in quasi-steady currents such as in rivers, estuaries or lakes, this limitation often only causes problems for extreme forcing conditions. In this case, velocity aliasing can be predicted and detected easily since the aliasing primarily occurs over a limited fraction of the profile. When the ACVP is mounted in a vertical downward looking direction as in Fig. 2a, the upper profile fraction (relative to the bed) is affected by aliasing because both the Doppler angle is smaller and the velocity is usually higher compared to the nearbed region. The aliased fraction of the velocity profile can be detected from the presence of an abrupt velocity change of amplitude equal to $\pm \nu_{j, \max }$. For this type of aliasing, the analysis of the Doppler phase or phase tracking methods (Rolland, 1994; Franca and Lemmin, 2006) allows the recovery of aliased velocity data. In oscillatory flows such as is observed under waves (Fig. 2a), the intra-wave velocity can vary over a much wider velocity range so that the velocity limits $\pm \nu_{j, \max }$ may be exceeded at each wave cycle, even in calm to moderate wave climates. Velocity aliasing is difficult to predict, detect and correct in this situation with standard de-aliasing methods. Also, the aliasing events can occur at different phases in wave cycles due to the typical irregular wave forcing found in nature.

A typical example is shown in Fig. 3 for a surf zone deployment with a JONSWAP sequence of irregular waves being skewed in both velocity and acceleration. The experiments were carried out at the UPC-CIEM wave channel at Barcelona, Spain (October 2008). The significant wave height, mean wave period and water depth at the wave paddle were set to $0.5 \mathrm{~m}, 5 \mathrm{~s}$ and $2 \mathrm{~m}$, respectively. The ACVP configuration used for this experiment is shown in Fig. 2b. A two component profiler is composed of two bistatic receivers aligned along the cross shore (i.e. wave propagation) direction and placed symmetrically relative to the vertical downward looking transceiver. Transceiver T was mounted $32 \mathrm{~cm}$ above the sand bed at a cross-shore position in the surf zone where the mean water depth at rest was equal to $65 \mathrm{~cm}$. The velocity profile was measured between the movable sand bed and the lowest wave trough. The ACVP parameters for this experiment were set to $1.25 \mathrm{MHz}$ for the carrier frequency, $P R F=1.6 \mathrm{KHz}$ and $N p p=32$ (See Eq. (5)) for the pulse-pair algorithm. This results in a velocity time resolution of $N p p / P R F=1 / 50 \mathrm{~s}$ and a maximal Doppler frequency $f_{D, j \max }=P R F / 2=800 \mathrm{~Hz}$.

Fig. 3 represents the timeseries of Doppler frequency $f_{D, 2}$ measured $20 \mathrm{~cm}$ above the sand bed with offshore receiver $R_{2}$ (Fig. 2a). Aliased Doppler frequency sequences can be seen at $t=0.5 \mathrm{~s}, 4.5 \mathrm{~s}, 9.1 \mathrm{~s}, 9.2 \mathrm{~s}$, $11 \mathrm{~s}, 12.4 \mathrm{~s}$ and $20 \mathrm{~s}$. Under these flow conditions, a systematic application of a robust de-aliasing routine is necessary in order to obtain reliable velocity data.

The de-aliasing method consists of calculating the following terms from the Doppler frequency $f_{D, 2}$ :

$$
\begin{aligned}
& f_{D, 2}^{+}=\left\{\begin{array}{l}
f_{D, 2}-P R F \quad \text { if } \quad f_{D, 2}>0 \\
f_{D, 2}+P R F \quad \text { if } \quad f_{D, 2}<0
\end{array}\right. \\
& f_{D, 2}^{-}= \begin{cases}f_{D, 2}+P R F & \text { if } \quad f_{D, 2}>0 \\
f_{D, 2}-P R F & \text { if } \quad f_{D, 2}<0\end{cases}
\end{aligned}
$$

Fig. 4a represents the timeseries of the original Doppler frequency $f_{D, 2}$ (cross symbols), the modified Doppler frequencies $f_{D, 2}^{+}$(circles) and $f_{\bar{D}, 2}^{-}$(stars) obtained with Eq. (17), respectively. It can be seen that during the aliased velocity sequences, the modified Doppler frequencies $f_{D, 2}^{+}$or $f_{D, 2}^{-}$correspond to the unaliased velocity values depending on the Doppler frequency range. In particular, when the Doppler frequency is aliased over the range $[-P R F ;-P R F / 2[U] P R F / 2 ; P R F]$, for example at $t=0.5 \mathrm{~s} / 4.5 \mathrm{~s} / 9.1 \mathrm{~s} / 9.2 \mathrm{~s}$, the Doppler frequency $f_{D, 2}^{+}$ corresponds to the unaliased Doppler frequency value. When the Doppler frequencies lie in the interval $[-3 P R F / 2 ;-P R F[\cup] P R F ; 3 P R F /$ $2]$, the Doppler frequency $f_{D, 2}^{-}$corresponds to the unaliased Doppler frequencies. For the last case, an example can be seen over a short sequence at $\mathrm{t}=12.2 \mathrm{~s}$ in Fig. 4a where the star symbols correspond to the correct Doppler frequency values. In order to replace the aliased frequencies sequences of $f_{D, 2}$ by the correct values, the following derivatives are estimated:

$$
\delta f_{D, 2}=\left\{\begin{array}{l}
\frac{N_{p p}}{P R F} \frac{\partial f_{D, 2}\left(t_{i}\right)}{\partial t}=f_{D, 2}\left(t_{i+1}\right)-f_{D, 2}\left(t_{i}\right) \\
\frac{N_{p p}}{P R F} \frac{\partial f_{D, 2}^{+}\left(t_{i}\right)}{\partial t}=f_{D, 2}^{+}\left(t_{i+1}\right)-f_{D, 2}\left(t_{i}\right) \\
\frac{N_{p p}}{P R F} \frac{\partial f_{D, 2}^{-}\left(t_{i}\right)}{\partial t}=f_{D, 2}^{-}\left(t_{i+1}\right)-f_{D, 2}\left(t_{i}\right)
\end{array}\right.
$$

$\Delta t=N_{p p} / P R F$ and a first order finite difference scheme are used for the approximation of the time derivatives. These three terms are shown in Fig. 4b. Among the three time series, the unaliased timeserie corresponds to the one closest to zero. Using these terms, the de-aliased Doppler frequencies $f_{D, 2}^{c}$ can be generated automatically with

$$
f_{D, 2}^{c}= \begin{cases}f_{D, 2}^{+}\left(t_{i}\right) & \text { if } \min \left(\left|\delta f_{D, 2}\right|\right)=\frac{N_{p p}}{P R F}\left|\frac{\partial f_{D, 2}^{+}\left(t_{i}\right)}{\partial t}\right| \\ f_{D, 2}^{-}\left(t_{i}\right) & \text { if } \min \left(\left|\delta f_{D, 2}\right|\right)=\frac{N_{p p}}{P R F}\left|\frac{\partial f_{D, 2}^{-}\left(t_{i}\right)}{\partial t}\right| \\ f_{D, 2}\left(t_{i}\right) & \text { else }\end{cases}
$$

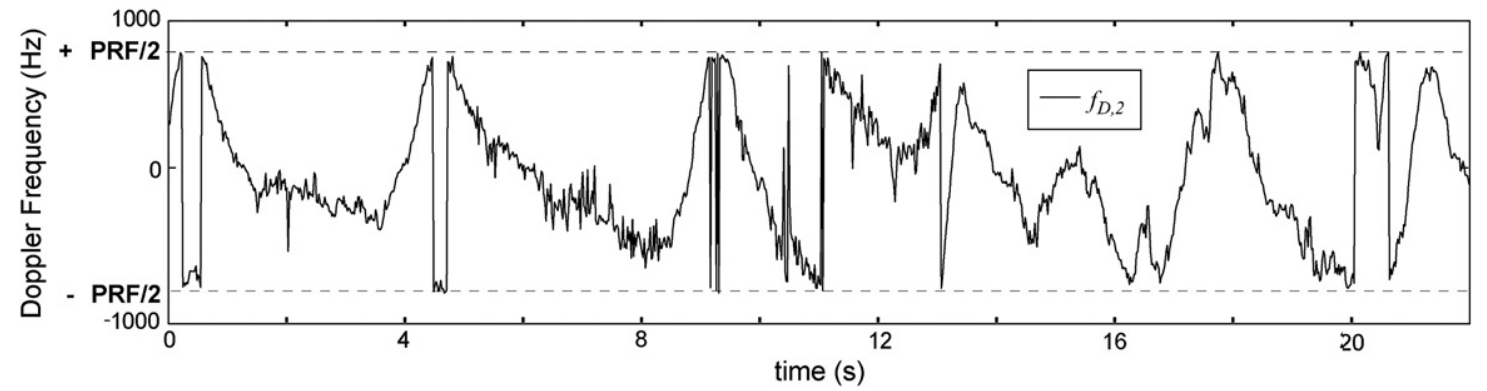

Fig. 3. Timeseries of Doppler frequency $f_{D, 2}$ affected by aliasing events. Measurements are carried out below irregular waves in the surfzone (see Fig. 2 a) $20 \mathrm{~cm}$ above the sand bed. 
a
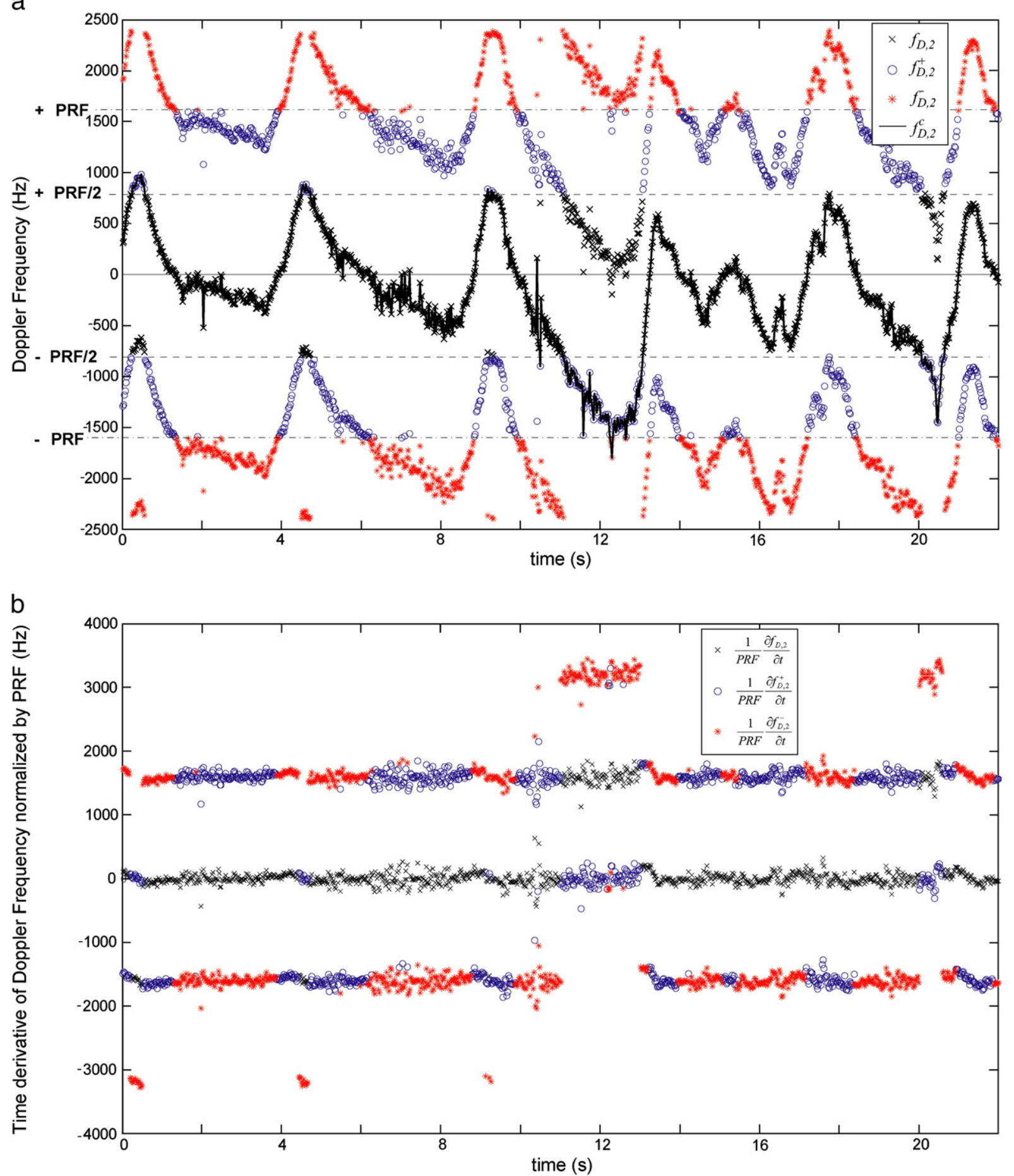

Fig. 4. (a) Timeseries of aliased Doppler frequency $f_{D, 2}$, modified Doppler frequencies $f_{D, 2}^{+}$and $f_{D, 2}^{-}$obtained with Eq. (17) and the corrected Doppler frequency obtained with Eq. (19). (b) Time derivative $\delta f_{D, 2}$ (Eq. (18)) used for the de-aliasing method.

The resulting de-aliased Doppler frequency $f_{D, 2}^{c}$ is represented in Fig. 4a by the thick black line.

In order to correct the reconstructed velocity components $u, v, w$ of Eq. (8), the present de-aliasing method must be applied separately to all the Doppler frequencies used in the geometrical recombination. Fig. 5 shows the end result of the de-aliasing method applied to the cross shore velocity component $u$.

Using this de-aliasing method, the resulting unambiguous Doppler frequency range of $f_{D, 2}^{c}$ is $[-3 P R F / 2 ; 3 P R F / 2]$ instead of $[-P R F / 2$; $P R F / 2$ ] for the original Doppler frequency $f_{D, 2}$. The relative range extension reaches $200 \%$. However, for the method to be used systematically as a de-aliasing algorithm, the following conditions apply: (a) the initial Doppler frequency $f_{D, 2}\left(t=t_{0}\right)$ cannot be aliased; (b) the time fluctuation due of the Doppler frequency (due to Doppler noise, spikes or physical turbulence) cannot exceed $\pm P R F / 2$.

The first condition can easily be met by taking an initial value of the velocity at which the wave velocity is known to be close to zero. For the second condition, an optimal $N_{p p}$ setting for the pulse-pair algorithm avoids the presence of strong Doppler noise levels. Nevertheless, when 


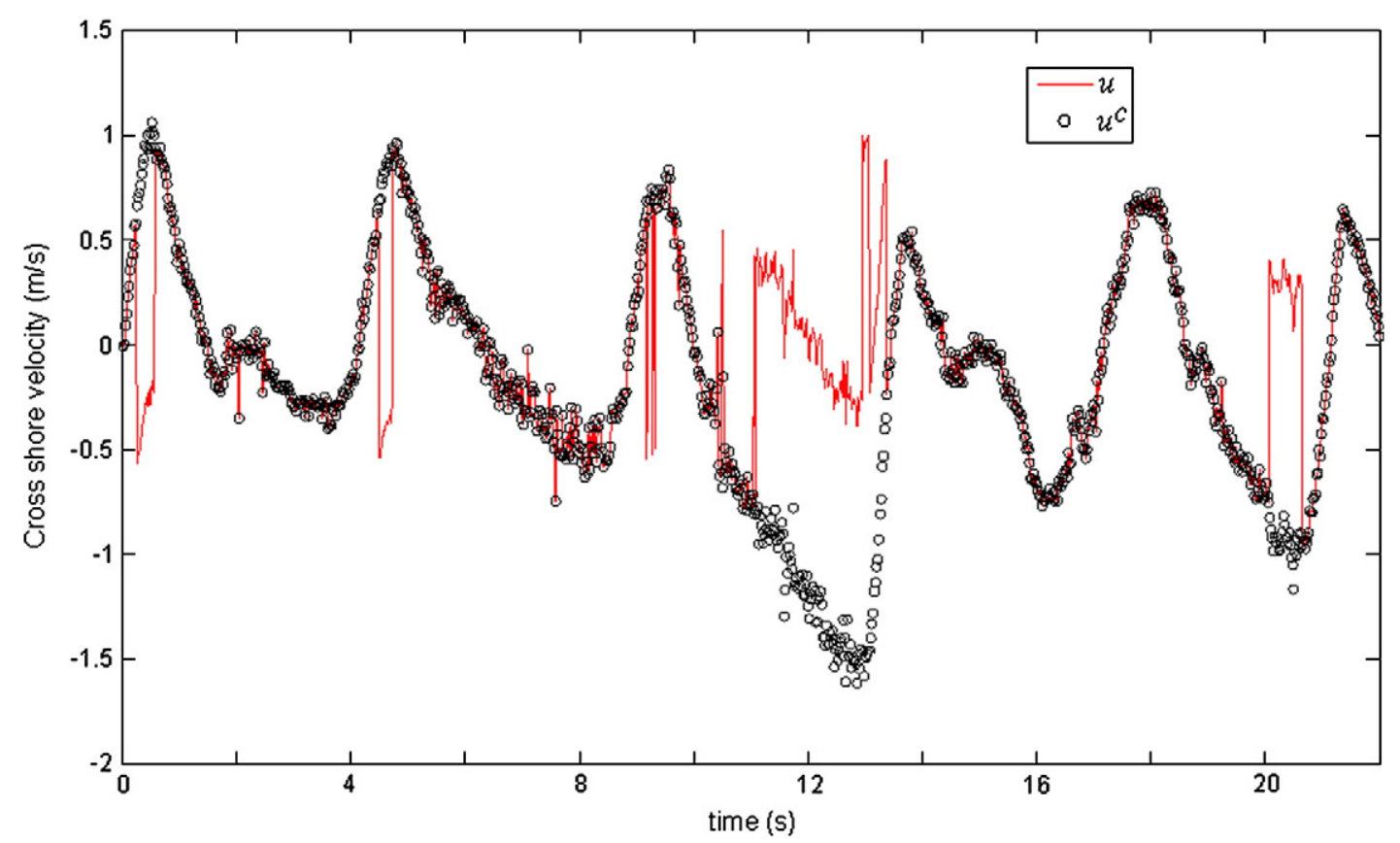

Fig. 5. Timeseries of corrected cross-shore velocity component, $u^{c}$ (circles), obtained after application of the de-aliasing procedure. The original time series, $u$, is shown by the red line.

spikes are present (usually attributed to a lack of seeding material or entrainment of large air bubbles), it is recommended to first run a despiking algorithm (Goring and Nikora, 2002) before the de-aliasing algorithm.

\section{Improvements in sediment concentration profiling across benthic suspension flows: a dual-frequency inversion method}

Thorne and Hanes (2002) concluded in their review on ABS performances for sediment transport studies that the inversion of the backscattered acoustic intensity into sediment concentration is subject to instabilities when the commonly used implicit iterative and explicit approaches are applied in case of non negligible sediment attenuation (Eq. (12) becomes implicit). This is particularly true across the nearbed region where high concentration values and gradients along the profile are seen. For example under oscillatory flows, the concentration can reach values of $0\left(10-100 \mathrm{~kg} / \mathrm{m}^{3}\right)$ in a layer close to the bed and can vary in time by one to two orders of magnitudes within the wave period. In these situations, the use of the standard inversion method requires careful handling to avoid inversion instabilities.

The purpose of this section is to present a novel acoustic inversion method that eliminates the inversion instabilities due to the implicit form of Eq. (12). It also offers advantages to sedimentologists using acoustics as a measuring tool, because it is a simpler approach than those presently available for extracting sediment concentration profiles from acoustic backscattered data.

In the following, we will first discuss the origin of the inversion instabilities for the two most commonly used methods known, the iterative implicit and the explicit inversion methods. Subsequently a dual-frequency inversion method will be proposed for a comparative analysis to the two previous methods. An experimental validation of the results is carried out in a sand suspension flow generated in an oscillating grid tank. This installation allowed setting the sediment concentration accurately under well controlled hydrodynamic conditions and for a wide range of mean concentration values by changing the oscillating grid frequency.
Starting from Eq. (12), we define the following variable:

$$
J_{l}=\frac{I_{l}}{A_{l} A_{s}}=C_{l} \exp \left(-4 \int_{0}^{\hat{r}} \zeta_{s} C d \hat{r}\right) \quad \text { where }\left\{\begin{array}{l}
I_{l}=I_{m o}\left(r=\frac{\tau c}{2} l\right) \\
A_{l}=A_{m o}\left(r=\frac{\tau c}{2} l\right)
\end{array}\right.
$$

$l$ is the gate index located at $r=\frac{\tau c}{2} l$. The names of the variables $I_{m o}$, $A_{m o}$ have been changed for simplicity in the writing. As previously stated, $A_{l}$ is the range dependent parameter including the hardware characteristics and the water absorption effect. $A_{s}$ is the acoustic backscattering parameter discussed in Section 3 and $J_{l}$ is the local sediment mass concentration affected by the non-linear sediment attenuation due to the presence of suspended sand along the travel path of the acoustic pulse train. When sediment attenuation effects are negligible, Eq. (20) becomes explicit and $J_{l}$ is simply equal to the local sediment concentration $C_{l}$. Calibration or semi-empirical relations are used for the determination of $A_{l}$ and $A_{s}$ as described above.

In the following, it is assumed that the parameters $A_{s}$ and $\zeta_{s}$ do not vary with distance from the transmitter, i.e. $n\left(a_{s}\right)$ is constant with height above the bed. This is usually valid across the highly concentrated nearbed region where inversion instabilities appear. The previous assumption means that no grain sorting occur in the highly turbulent nearbed flow region in which the Rouse number exceeds unity even for the largest size fraction of the grain size distribution.

\subsection{The iterative implicit inversion method}

The principle, performances and limitations of the iterative implicit inversion method have been discussed previously in the literature (Thorne et al., 1993; Thorne and Hardcastle, 1997). It relies on an iterative resolution of the implicit Eq. (20) due to the backscattered intensity attenuation caused by the suspended sediments along the travel path of the acoustic pulse. The concentration at the first gate is solved by iterating the relation $C_{1}^{n}=J_{1}^{0} \exp \left(4 \zeta_{S} C_{1}^{n-1}\right)$ over the iteration index $n$ until $C_{1}^{n+1}$ and $C_{1}^{n}$ converge to an arbitrarily 
fixed level of relative error. The initial value $J_{1}^{0}=C_{1}^{0}$ is first taken under the assumption of negligible attenuation. The concentration at the following gates can then be approximated as

$C_{l+1}=C_{l} \frac{J_{l+1}}{J_{l}} \exp \left(4 \zeta_{S} \int_{r_{l}}^{r_{l+1}} C(r) d r\right) \quad$ with $\quad l>1$

Where the attenuation integral in Eq. (21) between the two consecutive gates is approximated numerically by a rectangular discretization scheme as,

$C_{l+1}=C_{l} \frac{J_{l+1}}{J_{l}} \exp \left(4 \zeta_{S} C_{l} \Delta r\right)$

The gate-to-gate resolution of the last equation propagates cumulative errors from gate 1 to $l$ via the attenuation term. These errors are the consequences of the uncertainties in the inversion parameters. The sensitivity to these uncertainties increases significantly with gate index leading rapidly to infinitely high or negligible low concentration estimations when the sediment concentration is monotonic with distance from the transducer (usually the case for bed friction dominated suspension flows). This inversion instability is particularly problematic when the concentration approaches $1.0 \mathrm{~kg} / \mathrm{m}^{3}$ (typically the case in the nearbed region), the acoustic frequency is about $2 \mathrm{MHz}$ or higher and the mean sediment radius above $100 \mu \mathrm{m}$. It does not allow the iterative implicit approach to be used as a systematic acoustic inversion method.

\subsection{The explicit inversion method}

This method was proposed by Lee and Hanes (1995). It relies on the analytical solution of Eq. (12) taking its natural logarithm and after operating an adequate change of variable. The derivative with range $r$ can then be integrated and becomes

$$
C_{l}=\frac{J_{l}}{1-4 \zeta_{S} \int_{r_{1}}^{r_{l}} J(r) d r}
$$

It is assumed here that intensity attenuation due to sediments between the transducer and the first point in the profile is negligible, i.e. $C_{1}=J_{1}$. The integral of the normalized intensity $J(r)$ also has to be approximated by a discrete numerical scheme. Adopting a rectangular scheme as for the iterative implicit method, the local suspension concentration becomes

$$
C_{l}=\frac{J_{l}}{1-4 \zeta_{S} \Delta r \sum_{l=1}^{l-1} J_{l}}
$$

The problems here arise from the errors affecting the two terms in the denominator of Eq. (24). The value of 1 in the denominator is only valid under the assumption $C_{1}=J_{1}$. If this is not valid, the use of Eq. (24) will lead to underestimated or overestimated concentrations. The second source of errors in this method comes from the integral approximation of the second term in the denominator. The error in the approximation of normalized intensity $J(r)$ will induce a singularity in the concentration profile at the gate where the second term becomes close to unity. Before the singularity, the inverted concentration will be overestimated. After the singularity it will become negative before asymptotically reaching zero with distance $r$ from the transducer. Moreover, as for the iterative inversion method, the error propagates via the integral of the normalized intensity $J(r)$ between the first gate and gate $l$. Thus an error at any gate of the profile will affect the subsequent concentration estimates.

\subsection{A novel dual-frequency inversion method}

In order to eliminate the inversion instabilities due the gate propagating cumulative errors introduced by the uncertainties in the inversion parameters, Bricault (2006) proposes a dual-frequency inversion method. Starting from the following relation:

$\left(C_{l}\right)^{\zeta_{S k}-\zeta_{S i}}=\frac{\left(J_{i, l}\right)^{\zeta_{S k}}}{\left(J_{k, l}\right)^{\zeta_{S i}}}$

where $J_{i, l}$ and $J_{k, l}$ corresponds to the normalized intensities at frequencies $i$ and $k$, respectively, for $r=r_{1}$. The concentration profile can then be estimated from

$C_{l}=\left(J_{i, l}\right)^{\frac{1}{1-\zeta_{S i} / \zeta_{S k}}}\left(J_{k, l}\right)^{\frac{1}{1-\zeta_{S_{k}} / \zeta_{S_{i}}}}$

The advantages of this inversion are straightforward. First, no assumption on the sediment attenuation has to be taken at any gate in the profile. Secondly, the elimination of the attenuation integrals in Eq. (26) avoids the propagation of errors along the profile ensuring a gate independent sensitivity to the uncertainties in the inversion parameters.

The first condition for this dual-frequency method to work properly is that the smallest of the two acoustic frequencies lies within the Rayleigh or transition (equivalent to Mie scattering regime for electromagnetic waves) scattering regimes, i.e. below the geometric regime. This is the case when the parameter $k<a_{s}>$ is smaller than the critical value of $10 . k$ and $\left\langle a_{s}\right\rangle$ are the acoustic wavenumber and the mean radius of the suspended sediments, respectively. This condition can be respected easily for acoustic frequencies varying between $1 \mathrm{MHz}$ and $5 \mathrm{MHz}$ and for fine to coarse sand of mean radius up to $500 \mu \mathrm{m}$. Furthermore, due to the high frequency sensitivity of the attenuation coefficient $\zeta_{s} \approx f^{4}$ in the range $k a_{s}<1$ (roughly corresponding to the Rayleigh scattering regime), differences between both acoustic frequencies in the range of $100 \mathrm{KHz}$ induce $\zeta_{S i} / \zeta_{S k}$ ratios sufficiently different from unity. As a result, a single narrowband transducer can be used at both frequencies instead of two separate ones as in most of the multi-frequency ABS applications. This also offers the advantage of co-located sample volumes. In the present experiment, the dual-frequency method has been implemented with the frequency pair $1.67 \mathrm{MHz} / 2 \mathrm{MHz}$ using a unimodal broadsize sand suspension of mean diameter equal to $126 \mu \mathrm{m}$.

\subsection{A comparison between inversion methods}

Fig. 6 shows the installation of the ACVP in an Oscillating Grid Tank (OGT) at LEGI Grenoble, France. The horizontal grid was located $35 \mathrm{~cm}$ below the transmitting transducer and oscillated at a constant frequency of $5 \mathrm{~Hz}$. For a distance of about $8 \mathrm{~cm}$ above the average grid position, the flow is only subject to isotropic turbulent velocity fluctuation with negligible mean flow (Hurther and Lemmin, 2008). As a consequence, the suspension is in a steady equilibrium between the time-averaged sand settling flux (due to gravity) and the mean erosion flux (due to turbulence). In this experiment, the transmitter was aligned in the vertical direction normal to the grid plane. Therefore, the measured sand concentration profile was oriented vertically (Fig. 6).

The iterative, explicit and dual-frequency inversion methods have been applied to the acquired mean profiles of normalized intensity $J(r)$ as defined in Eq. (20). All system and acoustical parameters have been determined by calibration (Bricault, 2006). The sand concentration profiles obtained from the three acoustic inversions have been compared to pointwise measurements obtained with a calibrated 


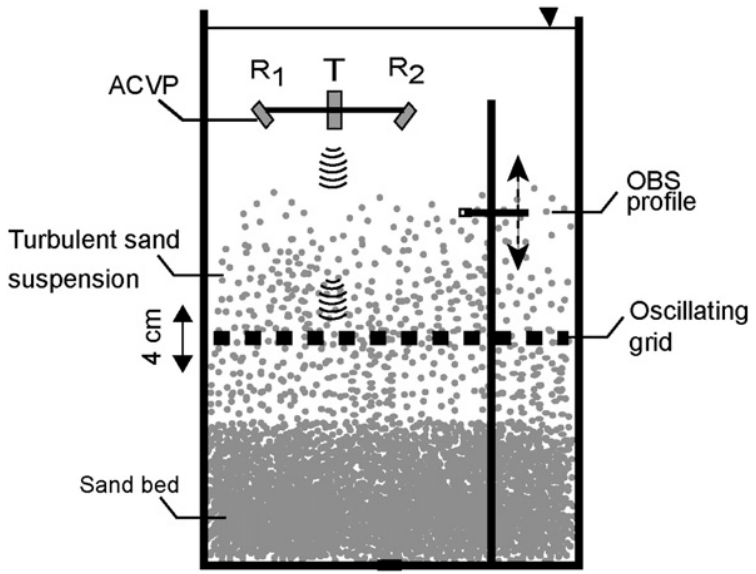

Fig. 6. Oscillating Grid Tank (OGT) used to generate a controlled sand suspension over a wide range of concentration values. The square meshed grid oscillates vertically at a constant frequency. The concentration profiles obtained with the three acoustic inversion methods (iterative, explicit and dual-frequency) were compared with OBS measurements.

Optical Backscattering System (OBS from D\&A instruments). The measurement uncertainty with this type of OBS and suspension is below $20 \%$

The iterative and explicit methods were implemented at an acoustic carrier frequency of $2 \mathrm{MHz}$. At this typical ABS frequency, acoustic attenuation due to sand suspension in the $1 \mathrm{~kg} / \mathrm{m}^{3}$ range generates inversion instabilities for a typical profiling range of tens of centimetres. The dual-frequency inversion uses the frequency pair $1.67 \mathrm{MHz} / 2 \mathrm{MHz}$ obtained quasi-simultaneously with the same emitting transducer. The same transducer has been used for the iterative and explicit inversions. The spatial resolution in the vertical direction is set to $3 \mathrm{~mm}$ with a Pulse Repetition Frequency of $1 \mathrm{kHz}$. A time averaging over 32 consecutive pulses was applied to the normalized intensity to ensure incoherent scattering properties. The resulting timeseries of normalized intensity was inverted using Eqs. (20), (24) and (26). Fig. 7 represents the time average of the concentration time series.

For the results obtained with the iterative and explicit methods (Eqs. (22) and (24)), the increasing divergence of the estimated concentration profiles from the OBS pointwise measurements is clear (Fig. 7). These trends confirm the presence of strong inversion

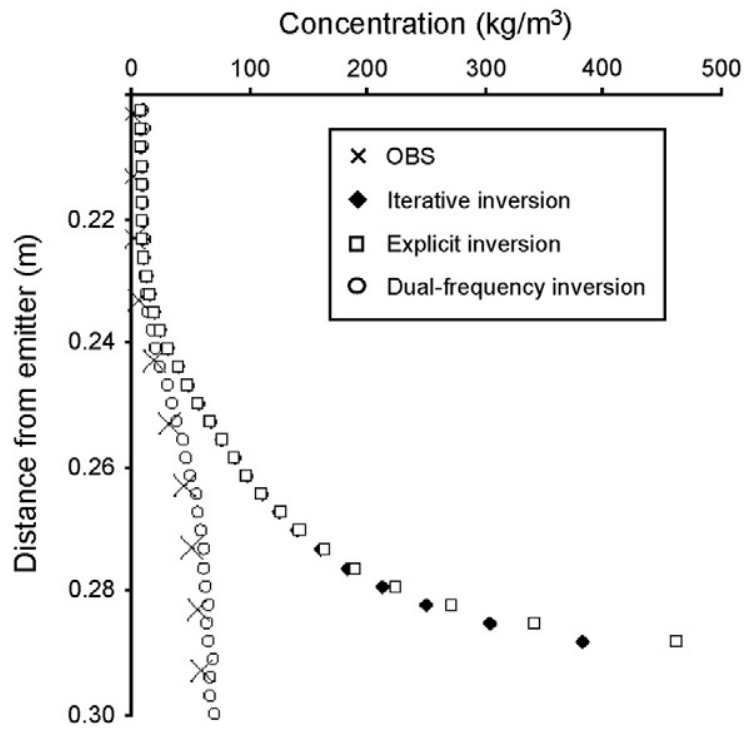

Fig. 7. Sand concentration profiles obtained with the three acoustic inversion methods and the pointwise OBS measurements. instabilities with the standard inversion methods when fine-scale profiling across high concentration suspensions is aimed. For both methods, the increase towards infinitely high concentration values with gate is the result of cumulative inversion errors associated with the monotonically increasing concentration profile.

The dual-frequency inversion results (Eq. (26)) are in better agreement with the OBS measurements over the entire profile. Both measurements indicate a sharp gradient region between $22 \mathrm{~cm}$ and $27 \mathrm{~cm}$ from the transducer followed by a fairly homogeneous concentration profile of about $60 \mathrm{~kg} / \mathrm{m}^{3}$. The OBS measurements support the proposition that if local errors are introduced in the dualfrequency inversion, they will not propagate along the profile via the attenuation term approximation, even when profiling through a strongly attenuating sand suspension. The relative errors obtained using the dual-frequency inversion were comparable with those obtained with the point measurements of the OBS.

\section{Application to sediment flux profiling above a rippled sand bed}

The present section aims to demonstrate the capability of the ACVP to make simultaneous co-located fine-scale profile measurements of velocity and sediment concentration by applying the previous system improvements. For this purpose, the ACVP was deployed in the Barcelona wave flume at a location where sand ripples formed under the action of a regular wave forcing (see Fig. 2a). The capability of the ACVP to obtain velocity, concentration and sediment flux profiles with sufficient temporal-spatial resolution to study sediment transport processes over a rippled bed is considered here.

Ripple bed vortex entrainment has been shown to play a significant role in the entrainment of sand from the bed into the water column across the coastal region (Bijker et al., 1976; Nakato et al., 1977; DuToit and Sleath, 1981; Nielsen, 1981; Fredsøe et al., 1999; Nichols and Foster, 2007; VanderWerf et al., 2007; Austin and Masselink, 2008; Thorne et al., 2009). Therefore the mechanism of sand entrainment in the ripple regime is under study owing to its importance in sediment transport under waves and combined waves and currents. However, it has proven difficult to obtain contemporaneous collocated suspended concentration and flow data, with sufficient spatio-temporal resolution and accuracy, to clearly establish the details of the vortex entrainment processes. Here we illustrate the performance of the ACVP in the rippled bed regime.

The wave conditions in the deep water region of the Barcelona wave channel ( $100 \mathrm{~m}$ long, $3 \mathrm{~m}$ wide and $5 \mathrm{~m}$ deep) were set to $0.5 \mathrm{~m}, 6.5 \mathrm{~s}$ and $1.9 \mathrm{~m}$ for the wave height, the wave period and the water depth, respectively. The sediment bed was composed of sieved sand grains of $d_{50}=250 \mu \mathrm{m}$. Fig. 8a represents the time evolution of the sand bed measured below the transmitter of the ACVP. It shows the presence of a ripple migrating onshore with a height of $2 \mathrm{~cm}$. Considering the time of $575 \mathrm{~s}$ taken by the ripple to migrate over a cross shore distance of $20 \mathrm{~cm}$ (measured separately by an Acoustic Ripple Scanner), the onshore oriented ripple migration speed was about $2 \mathrm{~cm} / \mathrm{min}$.

Fig. 8b shows the phase averaged cross-shore velocity (averaged over five consecutive waves) on the leeside of the ripple (onshore ripple face) measured with the ACVP at a distance of $2 \mathrm{~cm}$ and $20 \mathrm{~cm}$ above the sand bed at the location notified by the black circle in Fig. 8a. Fig. $8 \mathrm{c}$ presents the corresponding colour plot for the velocity profiles. It can be seen that the velocity is vertically homogeneous for $z>10 \mathrm{~cm}$ at any $t / T$ value. The velocity at $20 \mathrm{~cm}$ represents the wave velocity cycle outside the nearbed region influenced by the ripple. Focussing back onto Fig. $8 \mathrm{~b}$, it can be seen that between $t / T=0.35$ and 0.40 , the velocities at $2 \mathrm{~cm}$ and $20 \mathrm{~cm}$ above the bed have opposite signs. In particular, the velocity field outside the bedform influenced region remains positive (i.e. in onshore direction) during the wave crest and negative during the wave trough. However, the nearbed velocity, $\mathrm{z}<10 \mathrm{~cm}$, reverses before the wave crest-trough flow reversal, inducing a region of sharp flow gradient between $t / T=0.35$ and 0.40 . Within this 
a

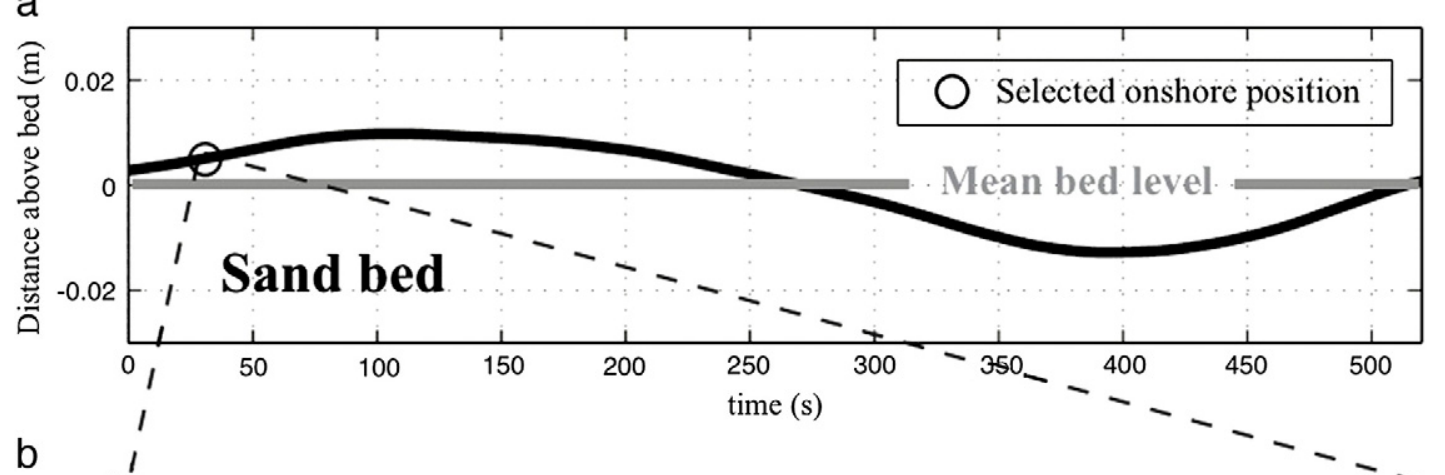

b

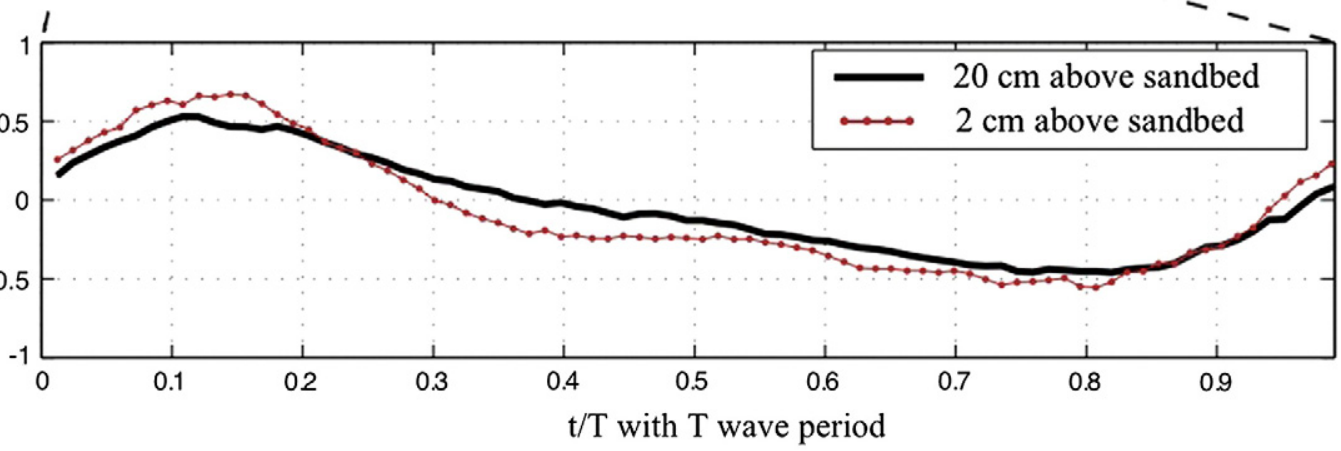

C

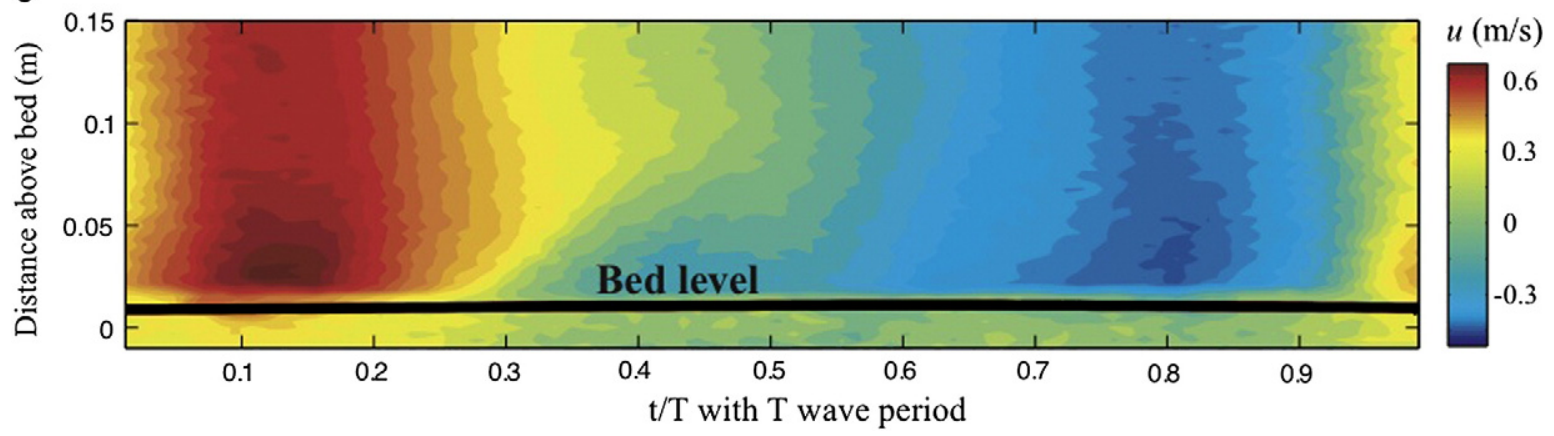

Fig. 8. ACVP measurements above an onshore migrating ripple. a) Ripple profile with time beneath ACVP (the circle marks the selected lee-side measurement location for Fig. 8b and c), b) measurements at the lee-side of the ripple side (marked by the black circle in a) of the cross shore wave velocity with intrawave time t/T, at $0.20 \mathrm{~m}$ (black) and $0.02 \mathrm{~m}$ (red dotted) above the sand bed, and c) colour map of the cross shore velocity profiles with intrawave time. The thick black line represents the bed position detected by the ACVP.

time period, the boundary between the negative and positive velocity regions in Fig. $8 \mathrm{c}$ is seen to evolve from $\mathrm{z}=0$ to $\mathrm{z}=10 \mathrm{~cm}$. When the corresponding 2D velocity vector field is generated, as shown in Fig. 9a, this boundary is seen to be associated with high transverse clockwise vorticity which is indicative of the presence of a vortex type flow structure at the end of the wave crest period (or leeside period). The absence in Fig. 9a of a similar flow detachment event (with counterclockwise direction) during the wave trough period (stoss side period), strongly suggests the presence of a leeside induced vortex shedding process.

In order to analyse the effect on sediment transport, Fig. 9b shows the sediment concentration profiles calculated with the dual-frequency inversion method described earlier. It can be seen that the flow regime with high values of suspended sediment concentration correspond well with the high vorticity region on the ripple leeside. Values as high as $4 \mathrm{~g} / \mathrm{l}$ were measured at a distance of up to $10 \mathrm{~cm}$ above the bed. Finally, it can be deduced from Fig. $9 \mathrm{c}$ that this region of high suspended sediment concentration is due to local sediment entrainment, since high values of sand erosion fluxes were also measured.

\section{Conclusions}

The ACVP presented here is the result of a joint development effort between three partners within the European project HYDRALAB III-
SANDS. The resulting system allows for a co-located (i.e. in the same insonified water column), quasi-instantaneous profiling of the suspended sand concentration and the multi-component velocity field for the measurement of sand fluxes at intrawave scales across the benthic flow region. Compared to previously developed systems (Shen and Lemmin, 1999; Smyth et al., 2002), the performance in terms of spatio-temporal resolution, velocity, concentration and sediment flux measurements, have been improved due to its multifrequency capabilities. This is achieved by using the same set of transducers in a frequency range between 1 and $2 \mathrm{MHz}$.

In particular, the well known unambiguous pulse-coherent velocity range has been extended by $200 \%$ using a simple, versatile and robust de-aliasing technique that can be applied systematically to the measured Doppler frequencies. As a result, the velocity can be dealiased under steady and oscillatory flows with important velocity variations as long as the turbulent fluctuations of the Doppler frequencies do not exceed the value $\pm P R F / 2$. The advantage of this method over the well-known dual-PRF dealiasing technique (Sirmans et al., 1976; Holleman and Beekhuis, 2003; Joe and May, 2003) is the absence of any specific hardware modifications in the system since the method relies on a software procedure of the acquired Doppler frequencies. The method has been tested in the challenging bathymetric wave breaking zone obtained with an irregular JONSWAP wave field in the wave channel of UPC-CIEM, Barcelona. The velocity 
a

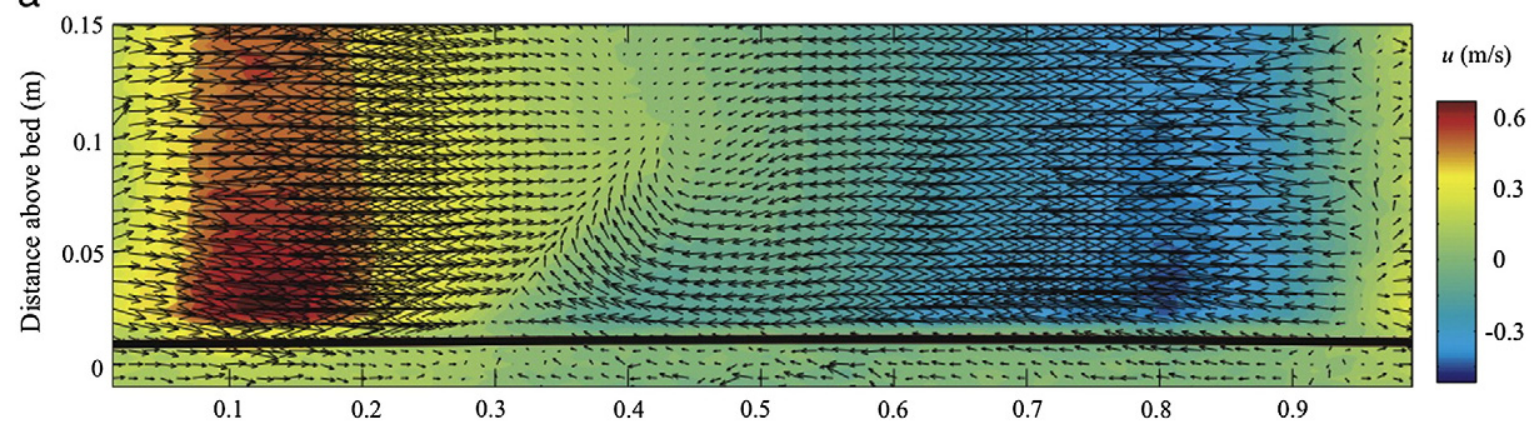

b

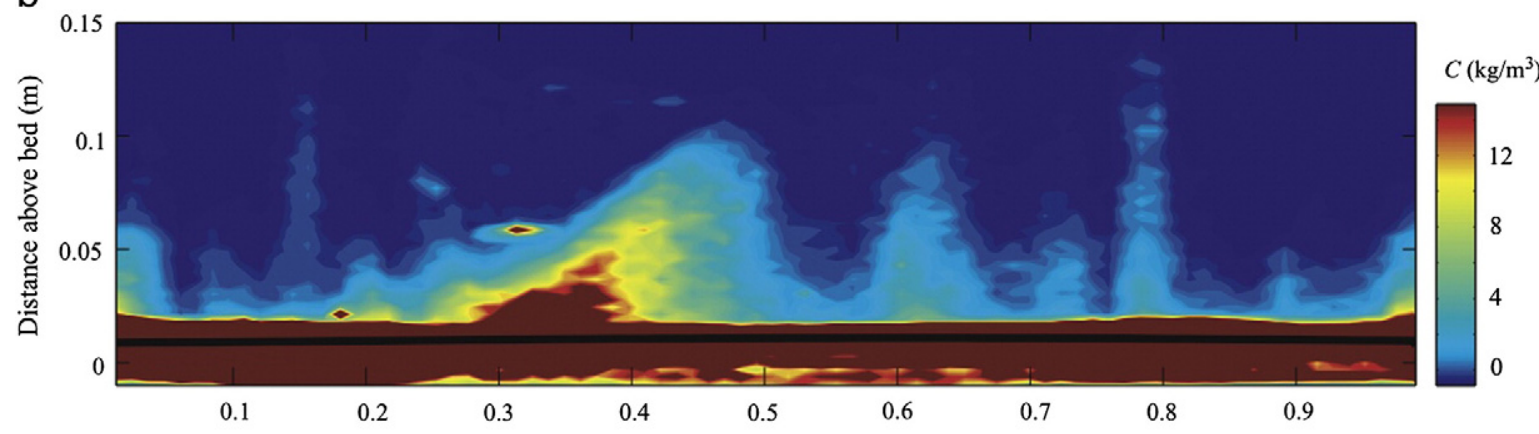

C

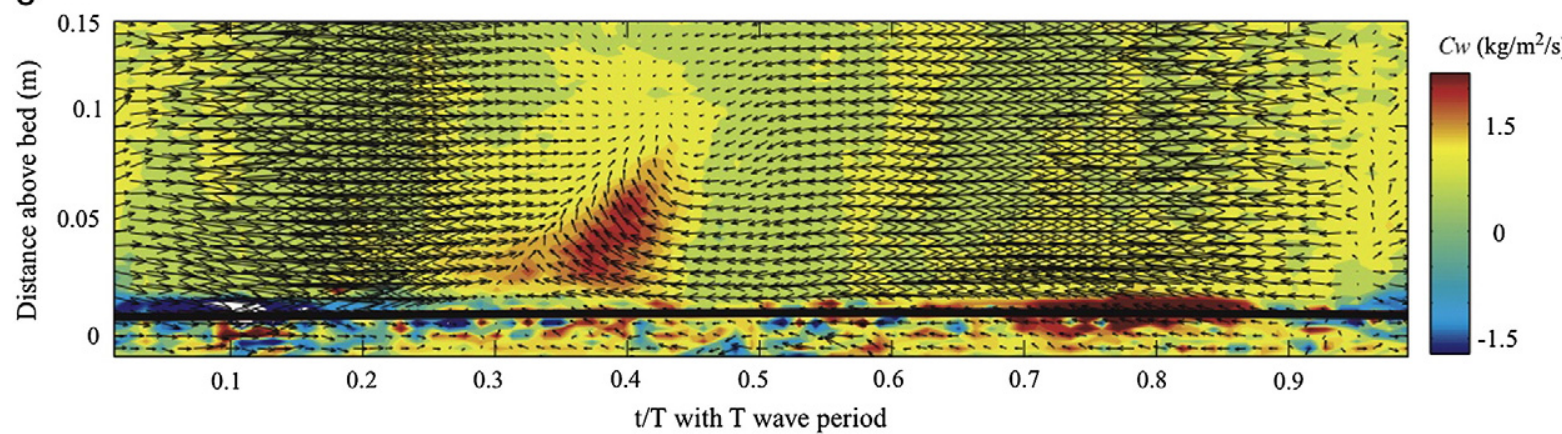

Fig. 9. a) Lee-side measurements (marked by the red circle in Fig. 8a) of the cross shore and 2D vector flow fields, b) The suspended sand concentration field, and c) The vertical sediment flux and 2D vector flow fields. The thick black line represents the bed position detected by the ACVP at the time marked by the circle in Fig. 8a.

timeseries sampled at a rate of $50 \mathrm{~Hz}$ could be de-aliased systematically under waves with important turbulence levels, strong acceleration and velocity skewness.

A novel dual-frequency inversion method has been proposed to eliminate the inversion instabilities obtained with the standard iterative implicit and explicit inversion methods (Thorne and Hanes, 2002). These instabilities lead to significant errors in the measured concentration profile across the benthic flow region when acoustic attenuation due to sediments occurs. Both the iterative implicit and the explicit methods are subject to gate-to-gate error propagation and accumulation along the profile reaching infinitely high concentration values. The proposed dual-frequency inversion method is locally independent in terms of error because the non-linear attenuation terms are eliminated in the inversion equation. It relies on the use of the backscattered intensities obtained at two slightly different acoustic frequencies from the same sand suspension. In order to work properly, the ratio between the attenuation constants at the two frequencies must be different from unity which means that the smallest frequency must lie within the Rayleigh scattering or transition regime outside the geometric regime. Due to the high frequency sensitivity of the attenuation coefficient for sand outside the geometric scattering regime, the method is stable for acoustic frequencies varying between 1 and $2 \mathrm{MHz}$. The concentration profiles obtained with the three inversion methods have been compared to pointwise OBS concentra- tion measurements obtained in an oscillating grid turbulence experiment with sand. The dual-frequency method gave results in good agreement with the local OBS data for sand concentration values of up to $60 \mathrm{~kg} / \mathrm{m}^{3}$ over a $15 \mathrm{~cm}$ thick sand suspension layer.

Making use of the previous system improvements, the capability of the ACVP to profile velocity, sand concentration and erosion fluxes above a rippled sand bed subject to vortex entrainment under waves was tested in a large scale wave flume experiment at UPC-CIEM Barcelona. At the lee-side of the ripple (i.e. the onshore face of the ripple), the presence of a clockwise vortex initiated before the wave flow reversal from onshore to offshore velocity is associated with a sand suspension cloud. This sediment cloud is seen to be correlated to an erosion flux event entraining sand over a vertical distance of roughly 4 times the ripple height above the bed. No such erosion flux event is seen to be correlated to a counter clockwise vortex during the opposite wave flow reversal from offshore to onshore velocity. This strongly supports the occurrence of leeside vortex entrainment. Whether the skewness of the waves in the shoaling zone generate an onshore oriented net suspended sediment transport as shown in VanderWerf et al. (2007) under a skewed water tunnel oscillatory flow, is currently under investigation in Hurther and Thorne (in press).

Measurements with the ACVP and video images have recently also been carried out at different locations across the wave breaking zone under irregular waves (Chassagneux and Hurther, 2009). The 
capabilities of the present ACVP for the study of cross shore sediment transport processes in this very energetic and challenging nearshore region will be further explored.

\section{Acknowledgements}

The present work was funded by the European project (FP6) Scaling Analysis and New instrumentation for mobile beD tests (SANDS) - within HYDRALAB III (European Commission contract No. 022441 (RII3)). The authors are grateful to Prof. A. Sanchez-Arcilla, Dr. I. Caceres, Dr R. D. Cooke, Dr B. D. Moate and F.X. Chassagneux for the helpful discussions and participation in the wave channel experience at UPC-CIEM, Barcelona (Oct. 2008).

\section{References}

Austin, J.M., Masselink, G., 2008. The effect of bedform dynamics on computing suspended sediment fluxes using optical backscatter sensors and current meters. Coastal Eng. 55, 251-260.

Betteridge, K.F.E., Thorne, P.D., Cooke, R.D., 2008. Calibrating multi-frequency acoustic backscatter systems for studying near-bed suspended sediment transport processes. Cont. Shelf Res. 28, 227-235.

Bijker, E.W., van Hijum, E., Vellinga, P., 1976. Sand transport by waves. Proc 15th ICCE, Honolulu, pp. 1149-1167.

Blanckaert, K., Lemmin, U., 2006. Means of noise reduction in acoustic turbulence measurements. J. Hydraul. Res. 44, 1-37.

Bricault, M., 2006. Rétrodiffusion acoustique par une suspension en milieu turbulent: application à la mesure de concentration pour l'étude de processus hydrosédimentaires. PhD thesis, Grenoble Institut National Polytechnique.

Chassagneux, F.X., Hurther, D., 2009. Nearbed flow/sediment processes under irregular breaking waves. Proceedings of the 33rd IAHR Conference, Vancouver, Canada.

Du Toit, C.G., Sleath, J.F.A., 1981. Velocity measurements close to rippled beds in oscillatory flows. J. Fluid Mech. 112, 71-96.

Franca, M., Lemmin, U., 2006. Eliminating velocity aliasing in acoustic Doppler velocity profiler data. Meas. Sci. Technol. 17, 313-322.

Fredsøe, J., Andersen, K.H., Sumer, B.M., 1999. Wave plus current over a ripple-covered bed. Coastal Eng. 38, 177-221.

Goring, D.G., Nikora, V.I., 2002. Despiking acoustic Doppler velocimeter data. J. Hydr. Eng. ASCE 128, 117-126.

Harris, K., Butman, B., Traykovski, P., 2003. Winter-time circulation and sediment transport in the Hudson Shelf Valley. Cont. Shelf Res. 23 (8), 801-820.

Hay, A.E., 1991. Sound scattering from a particle-laden turbulent jet. J. Acoust. Soc. Am. 90, 2055-2074

Hay, A.E., Mercer, D.G., 1985. On the theory of sound scattering and viscous absorption in aqueous suspension at medium and short wavelengths. J. Acoust. Soc. Am. 78, 1761-1771.

Holleman, I., Beekhuis, J., 2003. Analysis and correction of dual-PRF velocity data. J. Atmos. Oceanic Technol. 20, 443-453.

Hurther, D., Lemmin, U., 2001. A correction method of mean turbulence measurements with a 3D acoustic Doppler velocity profile. J. Atmos. Oceanic Technol. 18, 446-458.

Hurther, D., Lemmin, U., 2008. Improved turbulence profiling with field adapted acoustic Doppler velocimeters using a bi-frequency Doppler noise suppression method. J. Atmos. Oceanic Technol. 25 (2), 452-463.

Hurther, D., and Thorne P.D., Accepted for publication. Suspension and nearbed load sediment transport processes above a migrating sand rippled bed under shoaling waves. J. Geophys. Res.

Hurther, D., Lemmin, U., Terray, E.A., 2007a. Turbulent transport in the outer region of rough wall open-channel flows: the contribution of Large Coherent Shear Stress Structures (LC3S). J. Fluid Mech. 574, 465-493.
Hurther, D., Michallet, H., Gondran, X., 2007b. Turbulent measurements in the surf zone suspension. J. Coast. Res. SI50, 297-301.

Joe, P., May, P.T., 2003. Analysis correction of dual PRF velocity errors for operational Doppler weather radars. J. Atmos. Oceanic Technol. 20, 429-442.

Lee, T.H., Hanes, D.M., 1995. Direct inversion method to measure the concentration profile of suspended particles using backscattered sound. J. Geophys. Res. 100 (C2), 2649-2657.

Lhermitte, R., Serafin, R., 1984. Pulse-to-pulse coherent Doppler signal processing techniques. J. Atmos. Oceanic Technol. 1 (4), 293-308.

Mignot, E., Barthélemy, E., Hurther, D., 2009a. Double-averaging analysis and local flow characterization of near bed turbulence in gravel-be channel flows. J. Fluid Mech. 618, 279-303.

Mignot, E., Hurther, D., Barthélemy, E., 2009b. On the structure of shear stress and Turbulent Kinetic Energy (TKE) flux across the roughness layer of a gravel-bed channel flow. J. Fluid Mech. 638, 423-452.

Mignot, E., Hurther, D., Chassagneux, F.-X., Barnoud, J.-M., 2009c. A field study of the ripple vortex shedding process in the shoaling zone of a macro-tidal sandy beach. J. Coast. Res. SI 56.

Nakato, T., Locher, F.A., Glover, J.R., Kennedy, J.F., 1977. Wave entrainment of sediment from rippled beds. Proc ASCE, vol. 103, pp. 83-100. No WW1.

Nichols, C.S., Foster, D.L., 2007. Full-scale observations of wave-induced vortex generation over a rippled bed. J. Geophys. Res. 112, C10015.

Nielsen, P., 1981. Dynamics and geometry of wave generated ripples. J. Geophys. Res. $86,6467-6472$.

O'Hara Murray, R.B., Thorne, P.D., Hodgson, D.M., 2011. Intrawave observations of sediment entrainment processes above sand ripples under irregular waves. J. Geophys. Res 116, C01001, doi:10.1029/2010JC006216.

Rolland, T., 1994. Développement d'une instrumentation Doppler ultrasonore adaptée à l'étude hydraulique de la turbulence dans les canaux. PhD dissertation No 1281, Swiss Federal Institute of Technology (EPFL), Lausanne.

Shen, C., Lemmin, U., 1999. Application of an acoustic particle flux profiler in particleladen open-channel flow. J. Hydraul. Res. 37 (3), 407-419.

Sheng, J., Hay, A.E., 1988. An examination of the spherical scatterer approximation in aqueous suspensions of sand. J. Acoust. Soc. Am. 83, 598-610.

Sirmans, D., Zrnic, D.S., Baumgarner, W., 1976. Extension of maximum unambiguous Doppler velocity by use of two sampling rates. Preprints, 17th Conf. on Radar Meteorology, Seattle, WA: Amer. Meteor. Soc. , pp. 23-28.

Smyth, C.E., Zedel, L., Hay, A.E., 2002. Coherent Doppler profiler measurements of nearbed suspended sediment fluxes and the influence of bedforms. J. Geophys. Res. 107 (C8), 19.1-19.20.

Thorne, P.D., Buckingham, M.J., 2004. Measurements of scattering by suspensions of irregularly shaped sand particles and comparison with a single parameter modified sphere model. J. Acoust. Soc. Am. 116 (5), 2876-2889.

Thorne, P.D., Campbell, S.C., 1992. Backscattering by a suspension of spheres. J. Acoust. Soc. Am. 92, 978-986.

Thorne, P.D., Hanes, D.M., 2002. A review of acoustic measurement of small-scale sediment processes. Cont. Shelf Res. 22, 603-632.

Thorne, P.D., Hardcastle, P.J., 1997. Acoustic measurements of suspended sediments in turbulent currents and comparison with in-situ samples. J. Acoust. Soc. Am. 101 (5), 2603-2614.

Thorne, P.D., Meral, R., 2008. Formulations for the scattering properties of sandy sediments for use in the application of acoustics to sediment transport. J. Cont. Shelf Res. 28, 309-317.

Thorne, P.D., Hardcastle, P.J., Soulsby, R.L., 1993. Analysis of acoustic measurements of suspended sediments. J. Geophys. Res. 98 (C1), 899-910.

Thorne, P.D., Davies, J.S., Bell, P.S., 2009. Observations and analysis of sediment diffusivity profiles over sandy rippled beds under waves. J. Geophys. Res. 114, C02023, doi:10.1029/2008JC004944.

VanderWerf, J.J., Doucette, J.S., O'Donoghue, T., Ribberink, J.S., 2007. Detailed measurements of velocities and suspended sand concentrations over full-scale ripples in regular oscillatory flow. J. Geophys. Res. 112, F02012.

Zedel, L., Hay, A.E., 1999. A coherent Doppler profiler for high resolution particle velocimetry in the ocean: laboratory measurements of turbulence and particle flux. J. Atmos. Oceanic Technol. 16, 1102-1117. 